\title{
GLAD!
}

Revue sur le langage, le genre, les sexualités

11 | 2021

Archives, genre, sexualités, discours

\section{Archives, genre, sexualités, discours}

Introduction

Archives, Gender, Sexualities, Discourses

Mona Gérardin-Laverge, Magali Guaresi et Julie Abbou

\section{CpenEdition}

Journals

Édition électronique

URL : https://journals.openedition.org/glad/3638

DOI : $10.4000 /$ glad.3638

ISSN : 2551-0819

Éditeur

Association GSL

\section{Référence électronique}

Mona Gérardin-Laverge, Magali Guaresi et Julie Abbou, «Archives, genre, sexualités, discours », GLAD! [En ligne], 11 | 2021, mis en ligne le 20 décembre 2021, consulté le 29 janvier 2022. URL : http://

journals.openedition.org/glad/3638; DOI : https://doi.org/10.4000/glad.3638

Ce document a été généré automatiquement le 29 janvier 2022.

\section{cc) (†)}

La revue GLAD! est mise à disposition selon les termes de la Licence Creative Commons Attribution -

Pas d'Utilisation Commerciale - Pas de Modification 4.0 International. 


\title{
Archives, genre, sexualités, discours
}

\author{
Introduction
}

Archives, Gender, Sexualities, Discourses

Mona Gérardin-Laverge, Magali Guaresi et Julie Abbou

1 La question des archives, et des voix qu'elles font entendre, constitue un enjeu fondamental pour les luttes et les théories féministes. En effet, l'exclusion des femmes de l'espace public et des délibérations politiques ainsi que leur assignation à la sphère domestique et familiale sont allées de pair avec l'invisibilisation de leurs rôles politique et historique.

2 «Nous qui sommes sans passé, les femmes, nous qui n'avons pas d'histoire » chante l'Hymne des femmes: les mobilisations féministes des années 1970 contestent ensemble l'exclusion politique des femmes, la séparation public/privé et l'écriture de l'histoire. Dans le bouillonnement intense de ces années naissent des travaux pionniers en histoire des femmes, comme ceux de Michelle Perrot, qui cherchent à faire « reculer le silence, parfois si intense, qu'on a pu se demander: "Une histoire des femmes est-elle possible?" » (2001: v). Ces historien'ne's mettent au jour les mécanismes d'invisibilisation et de réduction au silence des femmes au moment de la production et de la conservation des archives : « la manière dont les sources sont constituées intègre l'inégalité sexuelle et la marginalisation ou dévalorisation des activités féminines. Ce défaut d'enregistrement primaire est aggravé par un déficit de conservation des traces » (ibid. : iv), que ce soit dans les archives publiques ou dans les archives privées conservées dans les grands dépôts publics, qui sont les archives des "grands hommes ». Et les archives familiales, les sources privées et l'écriture de l'intime, qui pourraient combler certaines de ces lacunes, ont longtemps été négligées par les historien-ne·s (ibid.).

3 Le constat que les femmes ont été effacées de l'histoire a poussé les militantes féministes, dès les années 1970, à produire leurs propres traces pour garder la mémoire et écrire l'histoire de leurs combats: elles filment leurs assemblées et leurs manifestations, photographient leurs compagnes de lutte, témoignent de leurs mobilisations et de leurs actions pour lutter contre leur invisibilisation, et écrivent 
l'histoire des féminismes (Pavard et al. 2020). Si le manque de temps, de lieux et de "réflexe archivistique » conduit de nombreux groupes militants à conserver très peu de traces de leurs activités, des associations et des collectifs féministes et/ou LGBTQIA+ se sont engagés depuis plusieurs décennies dans un travail de collecte, de conservation et de valorisation des archives de leurs mouvements (l'association Archives du féminisme, les Archives Recherches et Cultures Lesbiennes, le Centre audiovisuel Simone de Beauvoir, le Collectif Archives LGBTQI, l'association Mémoires des Sexualités, etc.).

4 Ces initiatives peuvent être en lien avec le monde académique, où des spécialistes de l'histoire des femmes, des sexualités et des féminismes réalisent un important travail pour collecter des archives et les rendre accessibles aux chercheurees, comme le fait notamment le Guide des sources de l'histoire du féminisme réalisé sous la direction de Christine Bard, Annie Metz et Valérie Neveu (2006). Comme en attestent plusieurs colloques (« Mémoires et transmission des archives lesbiennes et féministes » en 2016 à Paris, «Les féministes et leurs archives » en 2018 à Angers, «Archives du genre, genre des archives » à l'IMEC en 2019, " "Nos luttes changent la vie entière" : 50 ans de MLF » en 2020 à Toulouse, etc.) et programmes de recherche (par ex. le programme « Genre et transmission. Pour une autre archéologie du genre " à l'Université Paris Lumières de 2016 à 2019), les archives et le geste archivistique en eux-mêmes, interrogés au prisme du genre, font l'objet d'un intérêt scientifique important. Dans le cadre de ces manifestations scientifiques, on fait resurgir des archives, on exhume des fonds privés, on constitue de nouvelles archives (notamment via les témoignages oraux), on réexamine, dans une perspective féministe et/ou queer, certains fonds ou certains parcours de vie, et on élabore de nouvelles généalogies féministes et queer. Il ne s'agit plus seulement de retrouver des archives de femmes, mais d'interroger la constitution des archives par les pratiques institutionnelles et militantes, par le regard et le discours historiques, depuis une perspective critique sur le genre, les sexualités, la classe et la race.

5 Ce numéro thématique de GLAD! consacré aux archives s'inscrit dans ce contexte, et vise à interroger plus particulièrement la construction discursive du genre dans et par les archives. L'idée sous-jacente est la suivante : l'archive ne saurait être considérée comme un médium transparent vers les hiérarchies de genre du passé. Au contraire, les archives contribuent à construire les réalités, passées et contemporaines, au fil de parcours discursifs complexes qu'il convient de regarder, d'interroger et d'interpréter.

\section{Les archives et les discours du genre}

\section{Archives, discours et histoire}

6 Après des travaux pionniers dans les années 1970/90, qui ont cherché à théoriser et expérimenter l'intrication des faits linguistiques et des faits historiques au sein des archives, Jacques Guilhaumou reformule le concept d'« évènement discursif » en 2006. L'historien rappelle que l'évènement historique nous est transmis par le truchement du langage ou de l'archive. En cela, il est souvent un donné discursif, qu'on ne peut comprendre si l'on en ignore l'essence langagière. Selon Jacques Guilhaumou (2006: 48), « la dimension historique du fait social relève en grande part de ses conditions langagières d'apparition sans s'y confondre ». Il n'y a pas d'un côté le discours, et de 
l'autre la réalité, dont le premier rendrait compte de manière plus ou moins fidèle, mais une intrication forte entre les deux. Les évènements adviennent et sont appréhendés par les acteur-ice's à partir de catégories, de récits et de discours que les historien'ne's étudient a posteriori dans les sources textuelles.

7 Ce constat est évidemment valable pour l'histoire du genre et des sexualités. Discours d'une époque sur elle-même, les archives incarnent un pan de la réalité qu'elles configurent et actualisent dans un mouvement performatif, et qu'elles construisent dans sa dimension prescriptive. Et sans doute faut-il, pour les études de genre et sur les féminismes, postuler plus que jamais la consubstantialité des faits et des discours. Qu'est-ce que militer pour la cause féministe, si ce n'est déclarer, contester, argumenter, contredire, proposer, théoriser, délibérer...? Le cœur même de ces activités est d'ordre discursif. Ce sont par les mots, les formules et les slogans que se discutent les catégories, les hiérarchies, que se façonnent les identités et que s'élaborent les programmes de luttes.

\section{Matérialité des discours}

8 Système de règles, de codes, de représentations, le genre signifie la bipartition inégalitaire et sexuée des sociétés. En cela il fonctionne comme une grammaire, comme un « langage du politique » (Scott 1988, Achin \& Bargel 2013) pour imposer, légitimer et hiérarchiser les identités, les places et les relations des individuees et des groupes. Sans prétendre que le système genré soit exclusivement représentations langagières, il faut toutefois admettre que l'ordre du discours est l'une de ses meilleures traces ou encore l'un de ses facteurs les plus efficaces: c'est dans le langage d'une époque que s'inscrivent les rapports sociaux de sexes, que sont réifiées les catégories, que se perpétuent les antagonismes (Riot-Sarcey 2000).

9 Prendre acte du rôle du langage dans la construction du genre et dans les luttes féministes conduit à dépasser le dualisme de la matière (historique, économique ou corporelle) et du discours, pour interroger leurs interactions et mettre en lumière les effets matériels des discours. Dans différentes disciplines, un nombre croissant de travaux féministes cherchent ainsi à articuler une approche matérialiste et une approche post-structuraliste et queer ${ }^{1}$. La première est inspirée du matérialisme historique et explore les processus matériels et économiques qui structurent les rapports sociaux dans l'histoire ; la deuxième est inspirée du "tournant linguistique " et explore les processus discursifs de construction du genre. Les archives, en tant que discours matérialisés par excellence, radicalement dépendants de leur support, de leurs lieux et conditions de conservation, des pratiques sociales et institutionnelles et des processus idéologiques d'une époque, qui prennent sens dans et par le discours et l'interprétation historiques, permettent d'interroger l'intrication des dimensions matérielles et discursives des rapports sociaux de genre, et de documenter la construction discursive du genre. 


\title{
Comment constituer et traiter les archives ?
}

\author{
Discours de l'archive, discours sur l'archive
}

10 On pourra s'interroger sur la manière dont le discours historique produit le sens de l'archive, en fonction de parcours interprétatifs et herméneutiques. En ce sens, on n'épuise jamais vraiment une archive: on la relit toujours avec de nouvelles compétences interprétatives (et/ou techniques : quels sont les apports du numérique? Jusqu'à quel point les archives sont-elles des technologies ?). À partir de là, en quoi une perspective féministe et/ou queer sur le genre permet-elle de modifier le regard sur certaines archives et de renouveler leur interprétation? Dans quelle mesure permetelle de relire certains parcours de vie comme des parcours queer, certains évènements comme des manifestations de sexisme ou de misogynie, certaines pratiques comme des résistances, certains travaux historiques comme des gestes d'invisibilisation? Des relectures des chasses aux sorcières aux généalogies trans, de la mise au jour de l'agentivité des opprimée's dans la lignée des Cultural Studies aux projets d'« herstory », de nombreux'ses chercheure's ont entrepris de faire parler les archives pour lutter contre les silences de l'histoire. Ce numéro thématique de GLAD! est l'occasion d'interroger ensemble les discours des archives et les discours sur les archives au prisme du genre, pour voir en quoi cela permet de revisiter de manière critique tout à la fois l'évidence et la permanence des catégories majoritaires contemporaines, et l'invisibilité ou le silence des catégories minoritaires.

\section{Produire des archives}

11 La question de la transmission, de la citation et des mémoires féministes entre également dans ce champ d'interrogation. Comment les féministes interagissent-ielles avec leur histoire, avec leurs archives, avec les discours de luttes passées? Comment s'articulent les usages profanes, les usages militants, les usages académiques des archives? Comment les parcours individuels s'ancrent-ils dans des luttes collectives, comment le récit de soi s'articule-t-il au récit collectif? Ce numéro vise aussi à documenter différentes démarches et différents dispositifs de production d'archives dans une perspective militante, queer et/ou féministe. On peut penser notamment aux démarches consistant à recueillir des témoignages et des récits de vie afin de conserver la voix et les traces de groupes sociaux marginalisés. L'oral history, qui s'appuie sur ce type de démarche pour donner la parole aux acteurices, s'est principalement développée dans les pays anglophones. Si en France, l'effacement de l'historien·n'e qui se contenterait de "donner la parole» est considéré de manière plus critique (Descamps 2005), les sources orales ont progressivement gagné en légitimité depuis les années 1970/80, et la volonté de mettre à disposition des sources orales anime actuellement plusieurs projets (la commission audiovisuelle de l'association Archives du féminisme, le projet HERstory, etc.) qui réalisent des collectes de témoignages pour donner concrètement à entendre la multitude des voix qui font l'histoire des femmes et des luttes féministes.

12 Ces questions sont au cœur de ce nouveau numéro de GLAD! consacré aux liens entre archives, genre, sexualités et discours. Dans la rubrique "Recherches», on explore le rôle des archives dans la production de savoirs historiques depuis une perspective 
minoritaire, féministe, queer et/ou décoloniale. Certains articles proposent ainsi une relecture critique de certains corpus archivistiques au prisme du genre: Caroline Déodat interroge les répertoires raciaux de la créolité dans les poétiques du séga mauricien, et en propose une " autre histoire " à travers la notion de "polyphonie». Margot Giacinti mobilise le concept de "féminicide » afin de relire certaines archives judiciaires du XIX ${ }^{e}$ siècle dans une perspective féministe. Ruby Faure propose plusieurs lectures queer de lettres éditées par Michael Rosenfeld en interrogeant le rapport, à la fois personnel, scientifique et politique, du/de la chercheure aux archives.

13 D'autres textes explorent les enjeux de la production d'archives pour visibiliser certaines histoires, en lien avec des luttes actuelles. Delphine Frasch revient ainsi sur l'histoire féministe anglaise des années 1970, qui développe une " histoire par en bas " en inventant une nouvelle expérience, à la fois intime et collective, des archives. Mathilde Petit explore le rôle des archives dans la constitution et l'auto-définition de communautés militantes lesbiennes dans les années 1970 et 1980 aux États-Unis. Marine Gilis présente la collecte d'archives orales qu'elle a réalisée auprès de militantes des années 1970 du Mouvement des femmes en Bretagne et Pays de la Loire, et explique la nécessité pour l'historienne de s'engager elle-même dans la production d'archives, pour faire l'histoire de certains mouvements, lorsque les archives manquent.

14 Cette question de la production d'archives fait écho à plusieurs articles publiés dans la rubrique "Explorations». Ainsi, Maëva Ballon et Flaz Torres présentent le rôle politique des archives de leur activité militante féministe au sein d'un syndicat, dans la construction d'un rapport de force sur leur lieu de travail. Alexandre Klein et Gabriel Girard reviennent sur leur recherche sur les affiches, «archives oubliées » de la mobilisation militante, communautaire et associative, dans la lutte contre le sida à Montréal entre 1982 et 2012. Carolina Topini et Isabelle Salem Diego Sentis dévoilent l'actuel projet de collecte et de transmission d'archives « Nos lieux, nos fêtes, nos combats : notre histoire compte » du collectif « Notre histoire compte», qui a pour but de conserver, visibiliser et transmettre l'histoire des mouvements féministes et lesbiens à Genève. Marys Renné Hertiman analyse le travail réalisé par le groupe Les Bréchoises, qui cherche à développer les archives de femmes bédéastes pour rendre visible leur présence dans le neuvième art en France. Alain Giami élabore un essai autoethnographique sur sa propre démarche de dépôt d'archives de travail et d'enquête en vue de créer un centre d'archives sexologiques francophone dans un contexte académique. Enfin, Elie Grau propose dans ce numéro une traduction du «Manifeste de l'asexualité » de Lisa Orlando (1972), et analyse les enjeux politiques de la redécouverte de ce manifeste en 2019 par les communautés asexuelles en ligne.

D'autres contributions, en « Explorations » et en «Créations ", interrogent les archives existantes - et leurs possibles manques - au prisme du genre, de la race et des sexualités. Ainsi, Clémentine Rubio met au jour la quasi-absence de femmes dans les archives de la diplomatie linguistique, et s'applique à chercher des traces de leur rôle diplomatique, administratif et politique. Mathilde Sergent-Mirebault s'entretient avec deux archivistes du service d'Archives départementales d'Indre-et-Loire en charge du fonds Menie Grégoire, sur les enjeux de constitution et de conservation du fonds et l'intérêt de ces archives pour l'histoire des femmes et des sexualités. Sylvette Denèfle et Karine Lambert présentent une expérimentation collective, de huit artistes et scientifiques qui interrogent ensemble un même matériau archivistique, d'un féminicide perpétré à Toulon en 1847. En «Créations », Pensées sauvages publie une 
bande-dessinée intitulée "Déculonisons les archives ", issue d'une recherche dans les archives municipales sur les violences policières contre la population LGBT à Marseille dans les années 1930, qui questionne les manières dont on peut utiliser des archives institutionnelles produites dans un but répressif pour faire l'histoire des minorités.

Enfin, on pourra trouver dans ce numéro un article exploratoire Varia de Claire Gauzente, qui retrace une journée de «recherches-frictions» croisant travaux académiques et recherches artistiques sur la question des corps, ainsi que la neuvième chronique des «Genres récrits» de Daniel Elmiger, qui se demande "que faire du masculin à valeur générique?». En "Actualités », GLAD! publie une recension par Vanina Mozziconacci de la première anthologie de philosophie féministe en langue française, éditée par Manon Garcia.

\section{BIBLIOGRAPHIE}

ACHIN, Catherine \& BARGEL, Lucie. 2013. « "Montrez ce genre que je ne saurais voir". Genre, sexualité et institutions dans la présidentielle de 2012 ». Genre, Sexualité et Société, Hors-série 2.

BARD, Christine \& al. 2006. Guide des sources de l'histoire du féminisme : de la Révolution française à nos jours. Rennes : PUR.

BARD, Christine \& CHAPERON, Sylvie (éd.). 2017. Dictionnaire des féministes en France, XVIIIe-XXIe siècles. Paris : PUF.

BARD, Christine, BOIVINEAU, Pauline, CHARPENEL, Marion, GRAILLES, Bénédicte, LASSERRE, Audrey (éd.). À paraitre. Les Féministes et leurs Archives. Rennes : PUR.

CHARPENEL, Marion. 2014. Le privé est politique! Sociologie des mémoires féministes en France. Thèse de doctorat réalisée sous la direction de Marie-Claire Lavabre, Sciences-Po Paris.

DESCAMPS, Florence. 2005. L'historien, l'archiviste et le magnétophone. De la constitution de la source orale à son exploitation. Paris : Institut de la gestion publique et du développement économique, Comité pour l'histoire économique et financière de la France.

GUILHAUMOU, Jacques. 2006. Discours et événement. L'histoire langagière des concepts. Besançon :

Presses universitaires de Franche-Comté.

PAVARD, Bibia, ROCHEFORT, Florence et ZANCARINI-FOURNEL, Michelle. 2020. Ne nous libérez pas, on s'en charge. Une histoire des féminismes de 1789 à nos jours. Paris : La Découverte.

PERROT, Michelle. 2001. Les femmes ou Les silences de l'histoire. Paris : Flammarion.

PERROT, Michelle. 2006. Mon histoire des femmes. Paris : Seuil.

RIOT-SARCEY, Michèle. 2000. «L'historiographie et le concept de genre » Revue d'histoire moderne et contemporaine 47 (4) : 805-814.

SCOTT, Joan. 1988. « Genre : une catégorie utile d'analyse historique » Les Cahiers du Grif 37 (1) : 125-153. 


\section{NOTES}

1. Voir par exemple le colloque «Les féminismes matérialistes. Héritages épistémologiques et politiques, réappropriations contemporaines », 15-16 octobre 2021, Université Paris 1 PanthéonSorbonne [En ligne], consulté le 15/12/2021. URL: https://mediatheque.univ-paris1.fr/isjpsinstitut-des-sciences-juridique-et-philosophique-de-la-sorbonne-umr-8103/philosophiesfeministes-et-etudes-de-genre/.

INDEX

Thèmes : Recherches

Keywords : archives, history, feminism, LGBTQIA+

Mots-clés : archives, histoire, féminisme, LGBTQIA+ 